\title{
PERITONITIS IN CHILDREN. EPIDEMIOLOGICAL, CLINICAL AND THERAPEUTICAL STUDY
}

\author{
Adrian Surd ${ }^{1}$, Dan Gheban², Aurel Mironescu ${ }^{3}$, Cornel Aldea ${ }^{4}$, Horatiu Gocan ${ }^{1}$, Maria Forssén $^{1}$
}

Abstract

Introduction. The acute peritonitis is defined as the aggressive and diffuse inflammation of the peritoneum, of bacterial or chemical origin. Classification of peritonitis: primary, secondary (most common) and tertiary. Secondary peritonitis prognosis depends on: etiology, how fast the treatment is initiated, the age of the patient and the presence of associated pathologies. The objective of our study is to determine the frequency, epidemiological, clinical, paraclinical and etiological aspects of acute peritonitis and to evaluate the treatment quality in the Pediatric Surgery and Orthopedics Department of the Clinical Emergency Hospital for Children, Cluj-Napoca between October 2011 and October 2018.Material and methods: This study is a prospective one comprising 340 cases of acute localized or generalized peritonitis. The patients were hospitalized in the Pediatric Surgical Department of the Clinical Emergency Hospital for Children Cluj-Napoca, in a period of 4 years (October 2011 - October 2018). Inclusion criteria were represented by the clinical, imagistic and hematological documented peritonitis. Results: The most frequent cases of peritonitis were represented by appendicular peritonitis with an overwhelming proportion that agrees with literature data. On second place is the necrotizing enterocolitis. This is due to the addressability in the Pediatric Surgical Clinic on one hand and probably to the fact that premature patients are treated in county hospitals without logistic means or trained personnel. Posttraumatic peritonitis and peritonitis after peritoneal dialysis were on third place. Continuing our study we have observed that the post-operative peritonitis, pelviperitonitis, meconium peritonitis, peritonitis after Meckel diverticula, Crohn disease, intussusception, strangulated hernia, volvulus, perforated ulcer are much rarer. Based on where the lesion is located, the vast majority of peritonitis are originated from the submezocolical region and the most commonly perforated organ is the appendix. Discussion. In our study, the frequency of peritonitis was $12,4 \%$ of all surgical emergencies the average age was 7,8 years. Pain was the main symptom 191 of the patients having localized pain and 149 patients had diffuse pain. Vomiting was reported in $231(68 \%)$ patients and constipation or diarrhea in $83(24,4)$ patients. Regarding physical signs fever was present in 235 patient representing $69,11 \%$ of all patients. Abdominal ultrasound and plain abdominal X-ray were the main imagistic methods of investigations. Preoperative resuscitation is paramount in the treatment of peritonitis in children. Analgesia, nutritional support and sometimes even transfusion are critical in the treatment result improvement. Percutaneous drainage is an option in localized fluid collections but in generalized peritonitis abdominal lavage with warm saline a drainage are always indicated. In our study we noticed that most of the patients in which we used targeted lavage with a rectal tube, the rate of complications improved. Basically we used a small rectal tube (10-16 Fr) which we oriented in all intraabdominal zones with difficult access like pelvic regions, sub hepatic and sub diaphragmatic regions. We used a Guyon syringe to wash the abdominal cavity trough the rectal tube. This proved to be very efficient in avoiding infectious and obstructive complications. in intraabdominal infections. It is given daily in one dose and it is a good therapeutic option for patients that are allergic to penicillin and beta-lactam antibiotics. Moxifloxacin does not require dose adjustment in patients with impaired renal function, giving clinicians a monotherapeutic option in the treatment of complicated IAI. Conclusions: The patient with generalized acute peritonitis corresponds to a particular epidemiological profile related to: low socio-economical level, young age, male gender and pathological background.

Keywords: peritonitis, children, etiology

\section{Introduction}

The acute peritonitis is defined as the aggressive and diffuse inflammation of the peritoneum, of bacterial or chemical origin. Classification of peritonitis: primary, secondary (most common) and tertiary.

The peritonitis is a major emergency that needs hospitalization and urgent treatment. Although the mortality due to intra-abdominal infection is reduced, there is almost no progress in this domain recently. High rates of mortality are found in new born with necrotizing enterocolitis or peritonitis due to malformations of the gastrointestinal tract.

${ }^{1}$ Pediatric Surgery Department, Emergency Children's Hospital, Cluj-Napoca, Romania

${ }^{2}$ Head of Pathology Department, Emergency Children's Hospital, Cluj-Napoca, Romania

${ }^{3}$ Head of Pediatric Surgery Department, Children's Hospital, Brasov, Romania

${ }^{4}$ Pediatric Nephrology Department, Emergency Children's Hospital, Cluj-Napoca, Romania

E-mail: adisurd@yahoo.com,dgheban@gmail.com, aurel.mironescu@gmail.com,cornelaldea65@yahoo.com, horatiugocan@yahoo.com 
Secondary peritonitis prognosis depends on: etiology, how fast the treatment is initiated, the age of the patient and the presence of associated pathologies. High rates of mortality are found in postoperative peritonitis compared to secondary peritonitis (appendicitis, after Meckel diverticulitis etc.)[1].Peritonitis causes circulatory failure associated with shock, septic state with metabolic acidosis, multiple organ failure and ileus.

The treatment in this pathology involves resuscitation measures, surgical treatment of the source of intraperitoneal infection and adequate and prolonged antibiotic therapy.

The objective of our study is to determine the frequency, epidemiological, clinical, paraclinical and etiological aspects of acute peritonitis and to evaluate the treatment quality in the Pediatric Surgery and Orthopedics Section of the Clinical Emergency Hospital for Children, Cluj-Napoca between October 2011 and October 2018.

\section{Material and methods \\ Study type}

This study is a prospective one comprising 340 cases of acute localized or generalized peritonitis. The patients were hospitalized in the Pediatric Surgical Section of the Clinical Emergency Hospital for Children Cluj-Napoca, in a period of 4 years (October 2011 - October 2018).

\section{Study location}

The patients were admitted to the Pediatric Surgical Department either through the Emergency Reception Unit, or from other university clinics or county hospitals.

\section{Study population}

All patients between 0 and 18 years of age admitted in the Pediatric Surgical Section with the diagnosis of acute peritonitis based on clinical and paraclinical criteria were included in this study.

\section{Data}

To carry out the study, patient record were created (annex 1), where the investigator noted: identity, antecedents, clinical sings, paraclinical data, the received treatment and the outcome of the patients.

\section{Data analysis}

Statistical analysis was performed by simple descriptive methods consisting of calculating means and intervals for quantities variables, and percentages for qualitative variables.

\section{Ethical considerations}

The respect for the anonymity and the confidentiality of the patient data were both taken into account when the data was collected. All patients signed an informed consent.

\section{Results}

\section{Epidemiology}

a) Frequency: In our study, acute peritonitis accounted $12.4 \%$ of surgical abdominal emergencies, occupying second place after acute appendicitis. b) Sex: The male gender was predominant, with 201 cases, representing 59.11\% and 139 female cases, representing $40.89 \%$. The sex ratio was $1.44 \mathrm{M} / \mathrm{F}$ (Fig. 1)

c) Age: The age of the patients ranged from 1 day to 18 years, with an average of 7.8 years. The age group most commonly affected was between 6 and 12 years.

d) Antecedents: Chronic abdominal pain was detected in 28 patients, accounting $8.23 \%$ of the total patients and $13.40 \%$ of the 177 total patients with appendicular peritonitis.

Other antecedent conditions:

- 7 patients with congenital cardio-vascular disorders

- 5 patients with bronchopulmonary disease

- 18 patients with chronic kidney disease and peritoneal dialysis

- 4 patients with inflammatory bowel disease

- 2 diabetic treated patients

- 3 patients with gastric ulcer

- 1 case of treated pulmonary tuberculosis

- Facilitating factors:

Among the contributing factors are the living standard and leak of education. Other factors were abdominal trauma, low immunological status, cortisone treatment, appendicitis, diverticulitis, recent surgical treatment, peritoneal dialysis.

\section{Clinical data}

a) Functional signs:

Pain: a constant sign found at all patients. The pain was located in the epigastric or right iliac fossa in 191 patients, representing $56.18 \%$ of cases and generalized in 149 cases, representing $43.82 \%$.

Vomiting: variable in quantity and appearance. Was reported in 231 patients, representing $68 \%$ of the cases.

Transit disorders: Fecal transit disorders was present in 83 cases $(24.41 \%)$. Complete stopping of intestinal transit was reported in 31 cases, representing $9.11 \%$ of the cases. There were also 28 cases with rectal bleeding.

Associated clinical signs: besides pain, vomiting and transit disorders, other signs were detected in 43 patients, representing $12.64 \%$.

Time interval between onset of symptoms and consultation: it varied between 2 hours and 2 weeks, according to anamnesis.

b) Physical signs:

Fever: fever was reported in 235 cases, representing $69.11 \%$ of the cases.179 patients $(76.17 \%)$ were represented by appendicular peritonitis and 161 cases had a temperature below $38.5^{\circ} \mathrm{C}$. (Fig. 2)

Abdominal pain: abdominal contracture was reported in 181 cases $(53.23 \%)$. Generalized muscular defense was detected in 94 cases $(27.65 \%)$. Abdominal sensitivity was reported at 61 patients, representing $17.94 \%$ and in 4 cases $(1.18 \%)$ the abdominal palpation was without particular features (see table 1). 

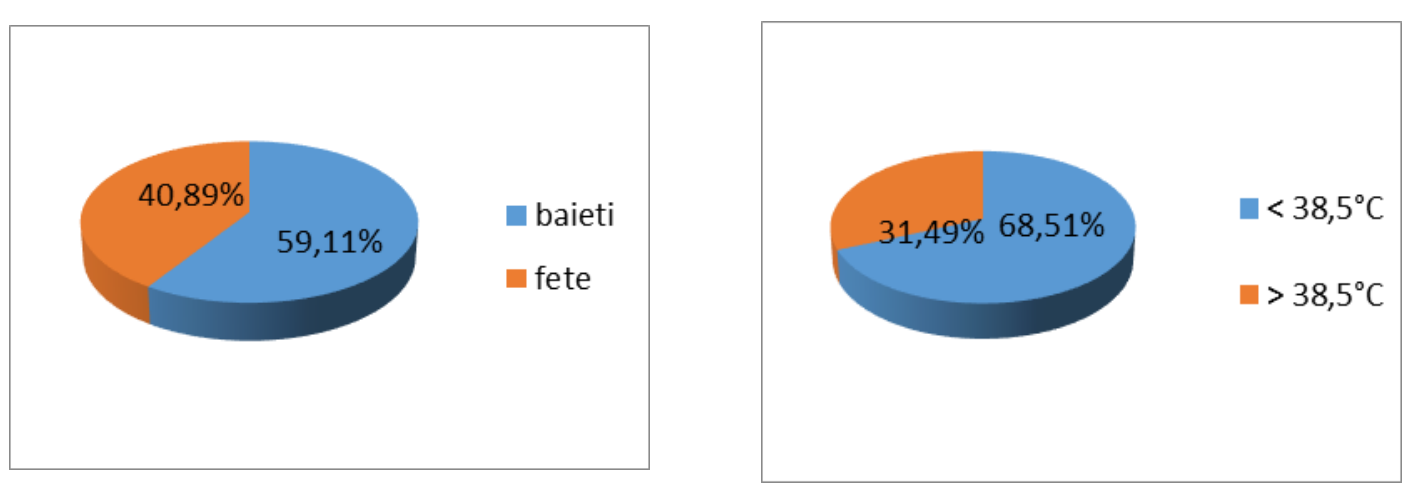

Fig. 1. Sex repartition

Fig. 2. Temperature $\left({ }^{\circ} \mathrm{C}\right)$ repartition

Table 1. Abdominal pain pattern

\begin{tabular}{|c|c|c|}
\hline Abdominal palpation & No of cases & Percentage \\
\hline Abdominal contracture & 181 & $53,23 \%$ \\
\hline General muscular defense & 94 & $27,65 \%$ \\
\hline Abdominal sensivity & 61 & $17,94 \%$ \\
\hline $\begin{array}{c}\text { Abdominal palpation } \\
\text { without particular features }\end{array}$ & 4 & $1,18 \%$ \\
\hline
\end{tabular}

Table2. Etiology of peritonitis

\begin{tabular}{|l|l|l|}
\hline Etiology & Number of cases & Percentage \\
\hline Appendicular peritonitis & 209 & $61,47 \%$ \\
\hline Necrotizing enterocolitis & 35 & $10,29 \%$ \\
\hline Post-operative peritonitis & 14 & $4,12 \%$ \\
\hline Post-traumatic peritonitis & 26 & $7,65 \%$ \\
\hline Peritonitis after peritoneal dialysis & 26 & $7,65 \%$ \\
\hline Peritonitis after intussusception & 3 & $0,88 \%$ \\
\hline Peritonitis after Meckel diverticulum & 4 & $1,18 \%$ \\
\hline Pelviperitonitis & 6 & $1,76 \%$ \\
\hline Peritonitis after perforated gastric ulcer & 3 & $0,88 \%$ \\
\hline Peritonitis after strangulated hernia & 2 & $0,59 \%$ \\
\hline Meconium peritonitis & 5 & $1,47 \%$ \\
\hline Peritonitis after Crohn disease & 4 & $1,18 \%$ \\
\hline Peritonitis after volvulus & 3 & $0,88 \%$ \\
\hline TOTAL & 340 & $100 \%$ \\
\hline
\end{tabular}




\section{Paraclinical data}

a) Abdominal X-Ray:

148 patients had an abdominal or thoraco-abdominal $\mathrm{X}$-Ray done. Pneumoperitoneum was detected in 43 cases, representing $29.05 \%$ of the patients. Hydroaeric levels was found in 32 cases $(21.62 \%)$ and intestinal pneumatosis was detected in 14 cases, representing $9.45 \%$ of the patients (all affected by necrotizing enterocolitis). In the remaining 59 patients, representing 39.86 of cases, the radiological examination showed no changes.

b) Abdominal ultrasonography:

Abdominal ultrasonography was performed in 324 cases, representing $95.29 \%$ of the total number of patients and at all patients with appendicular peritonitis. In 281 cases $(82.64 \%)$ this procedure detected variable peritoneal collections variable in localization and appearance. Of the patients with appendicular peritonitis, 130 cases presented intraabdominal collections, 28 patients showed suggestive signs of acute appendicitis, 12 patients had an abscess in the right iliac fossa and 7 cases were without particular ultrasonography changes.

c) Abdominal CT:

Only 12 patients with post-traumatic peritonitis benefited from an abdominal CT.

d) Hemoleucogram:

The hemoleucogram was performed in all patients. In 28 patients, representing $8,24 \%$ of the cases, the leukocyte formula was normal or had leukopenia. In 198 patients $(58,24 \%)$ the leukocyte number was between 11,000 and $15,000 / \mu 1$. 71 patients $(20,88 \%)$ had the number of leukocytes between 15,000 and $20,000 / \mu 1$. The other 43 patients $(12,65 \%)$ had leukocytosis with values over $20,000 / \mu 1$.

e) C-reactive protein:

CRP was performed in all patients. 20 patients $(5,88 \%)$ had values of under $0,5 \mathrm{mg} / \mathrm{dl}$. 81 patients $(23,82 \%)$ had values of the CRP between 0,5 and $5 \mathrm{mg} / \mathrm{dl}$. 123 patients(36,18\%) had values between 5 and $10 \mathrm{mg} / \mathrm{dl}$. The remaining $116(34,12 \%)$ patients had values of the CRP over $10 \mathrm{mg} / \mathrm{dl}$. As we will find during the CRP study, along with procalcitonin, the CRP has predictive value in terms of morbidity associated with peritonitis in infants. Procalcitonin was collected in 98 patients, representing $28.82 \%$ of the patients.

\section{Etiological data}

In our study, the diagnosis of peritonitis was based on anamnestic data, clinical and paraclinical data and on exploratory laparotomy.

The most frequent cases of peritonitis were represented by appendicular peritonitis with an overwhelming proportion that agrees with literature data. On second place is the necrotizing enterocolitis. This is due to the addressability in the Pediatric Surgical Clinic on one hand and probably to the fact that premature patients are treated in county hospitals without logistic means or trained personnel. Post-traumatic peritonitis and peritonitis after peritoneal dialysis were on third place. Continuing our study we have observed that the post-operative peritonitis, pelviperitonitis, meconium peritonitis, peritonitis after Meckel diverticula, Crohn disease, intussusception, strangulated hernia, volvulus, perforated ulcer are much rarer. Based on where the lesion is located, the vast majority of peritonitis are originated from the submezocolical region and the most commonly perforated organ is the appendix. (Table 2)

\section{Therapeutically approach}

a) Preoperative care:

Initial treatment consisted in peripheral venous catheterization for hydroelectrolytic and acid-base rebalancing. All newborns as well as patients requiring parenteral nutrition benefited from the installation of a central venous catheter. Patients that associated occlusive syndrome and those surgically treated immediately after admission in the hospital benefited from the installation of a naso-gastric probe.

b) Medical treatment:

Antibiotics: antibiotic treatment was initiated either preoperatively or at the induction of anesthesia, being continued between 5 to 21 days postoperatively.

Out of 340 patients, $79(23.24 \%)$ patients were treated with a single broad-spectrum microbial agent (betalactamase inhibitor), 91(26.76\%) patients had dual therapy (beta-lactamase inhibitor/cephalosporin and macrolide) and 170 patients $(50 \%)$ benefited from triple antibiotic combination (cefuroxime, clindamycin and metronidazole). All patients with antibiotic treatment that lasted more than 5 days have been also treated with an antifungal agent.

Antalgic treatment: all patients were given antalgic treatment during hospitalization and also at discharge (between 0 and 10 days).

Nutritional support: patients who were unable to feed in the first postoperative days benefited from parenteral nutrition, usually with Aminovem, together with glucose, electrolytes and vitamins. Probiotics (Lactobacillus and Bifidobacterium) were also given to newborns, especially at those with necrotizing enterocolitis.

Blood transfusion: patients with severe septic shock or associated hemorrhagic shock, as well as those with severe thrombocytopenia received whole blood, plasma or platet mass. A total of 31 patients $(9.12 \%)$ have had transfusions. c) Surgical treatment:

All patients from our study have undergone surgical treatment, except for the patients with peritonitis associated with peritoneal dialysis.

Peritonitis treatment:

Depending on the preoperative diagnosis, peritonitis treatment required surgical approach either through the right iliac fossa, or median approach if it was necessary to explore the whole peritoneal cavity. The peritoneal liquid was collected for laboratory samples and then evacuated, as well as abundant washing of the peritoneal cavity until clear fluid was highlighted was made. After the surgery, one or two peritoneal drainage tubes were installed. 
Table 3. Most common intraabdominal lesions

\begin{tabular}{|l|l|l|l|}
\hline Lesion & Number of cases & Percentage & Therapeutically gesture \\
\hline $\begin{array}{l}\text { Small intestine } \\
\text { rupture }\end{array}$ & 6 cases & $30 \%$ & $\begin{array}{l}\text { Intestinal suture/Bowel } \\
\text { segmental resection and } \\
\text { anastomosis }\end{array}$ \\
\hline Liver rupture & 3 cases & $15 \%$ & Hepatoraphy \\
\hline Splenic rupture & 6 cases & $30 \%$ & Splenoraphy/Splenectomy \\
\hline Pancreatic rupture & 3 cases & $15 \%$ & Drainage/Suture \\
\hline Renal rupture & 2 cases & $10 \%$ & $\begin{array}{l}\text { Suture of the kidney and } \\
\text { bassinet }\end{array}$ \\
\hline
\end{tabular}

Table 4. Patients outcome

\begin{tabular}{|c|c|c|c|}
\hline \multicolumn{2}{|l|}{ Evolution } & Number & Percentage \\
\hline \multicolumn{2}{|l|}{ Favorable } & 252 & $74,12 \%$ \\
\hline \multirow[t]{5}{*}{ Complications } & $\begin{array}{l}\text { Intraabdominal } \\
\text { abscess }\end{array}$ & 8 & $2,35 \%$ \\
\hline & $\begin{array}{l}\text { Deep-wound } \\
\text { infections }\end{array}$ & 16 & $4,70 \%$ \\
\hline & $\begin{array}{l}\text { Superficial wound } \\
\text { infections }\end{array}$ & 25 & $7,35 \%$ \\
\hline & $\begin{array}{l}\text { Sepsis with resistant } \\
\text { germs }\end{array}$ & 14 & $4,12 \%$ \\
\hline & Pneumopaty & 2 & $\mathbf{0 , 6 0}$ \\
\hline \multicolumn{2}{|l|}{ Mortality } & 23 & $6,76 \%$ \\
\hline \multicolumn{2}{|l|}{ Total } & 340 & $100 \%$ \\
\hline
\end{tabular}

Peritoneal fluid:

The peritoneal fluid was purulent in 281 cases, representing $82.65 \%$ of the patients. Hemoperitoneum was present in 24 cases $(7.06 \%)$. The visceral peritoneum was most often inflamed and false membranes were found in 168 patients, representing $49.41 \%$.

The digestive and bile fluid was found in 9, respectively 4 cases.

d) Etiological treatment:

Appendicular peritonitis: direct or retrograde appendectomy was performed at all patients with appendicular peritonitis. In 61 cases $(29.19 \%)$ out of 209 patients direct appendectomy was performed and retrograde appendectomy was performed at 148 patients (70.81\%). Also, lavage with minimum 21 of warm serum and Douglas or right latero-colical space drainage was performed.

Necrotizing enterocolitis: in 27 cases $(77.14 \%)$ out of 35 patients, exploratory laparotomy was performed and in
$8(22.86 \%)$ cases, percutaneous drainage of the peritoneal cavity. Of the patients with laparotomy, 6 patients underwent enteropathy and drainage, 19 underwent segmental resection of the intestine and ileostomy/colostomy and drainage and 2 patients underwent ileostomy and drainage.

Peritonitis through perforated ulcer: All patients underwent suture of the gastric wall and filling with omentum and drainage.

Peritonitis after Meckel diverticulum: Patients with peritonitis after Meckel diverticulum have had segmental ileum resection, lavage and drainage.

Post-traumatic peritonitis: of the post-traumatic peritonitis $20(76.92 \%)$ patients had closed abdominal trauma and 6 patients $(23.08 \%)$ had open abdominal trauma. Of the patients with open abdominal trauma, 4 cases had small bowel lesions, one patient had stomach 
lesion and other patient had a colon lesion. These patients underwent lesion sutures, lavage and drainage. (Table 3)

Pelviperitonitis: patients with pelviperitonitis due to piosalpinx have benefited for abscess evacuation and peritoneal drainage.

Postoperative peritonitis: in $10(71.43 \%)$ out of 14 patients the cause was the dehiscence of the ileo-colic, ileoileal or jejuno-ileal anastomosis. In 4 patients the cause of the peritonitis could not be detected. Peritonitis after intussusception, strangulated hernia or intestinal volvulus were treated with segmental resection of the ileon or ileon and right colon and ileo-ileal or ileo-colic anastomosis.

\section{Outcome}

a) Mortality:

In our study there were 23 deaths, representing $6.67 \%$ of the cases. 18 deaths occurred at infants with necrotizing enterocolitis, 1 death in a patient with meconium peritonitis and septic shock, 2 deaths in patients with postoperative peritonitis, septic shock and multiple organ failure and 2 deaths in polytrauma patients.

b) Morbidity:

Postoperative evolution was favorable in most cases. Postoperative complications occurred in 65 cases, representing $19.12 \%$ of the patients. 8 patients developed intraabdominal abscesses, 16 patients developed abdominal wall infections, 14 cases with septic state due to nosocomial infections and 2 patients presented pneumopathy. (Table 4)

c) Postoperative evolution:

252 patients, representing $74.12 \%$ of the cases had a good postoperative evolution, the intestinal transit resumed after an average of 3 days and the nasogastric probe (at those who needed installation) was suppressed in the 4-th day after surgery.

d) Hospitalization period:

The hospitalization period was between 5 and 27 days, with an average of 6 days of hospitalization.

e) Medium/long term outcome:

5 patients developed occlusive syndrome in 1 to 4 months after the surgery. 1 patient was treated conservatively with gas tube and nasogastric probe. In 4 patients surgery was performed with adesiolysis and clamp resection with favorable outcome.

\section{Discussion}

Peritonitis remains the main surgical abdominal emergency in children. Most of the children are diagnosed with some delay, hence the increased frequency of peritonitis. In our study, the frequency of peritonitis was $12,4 \%$ of all surgical emergencies the average age was 7,8 years. Pain was the main symptom 191 of the patients having localized pain and 149 patients had diffuse pain. Vomiting was reported in $231(68 \%)$ patients and constipation or diarrhea in $83(24,4)$ patients. Regarding physical signs fever was present in 235 patient representing $69,11 \%$ of all patients. Abdominal ultrasound and plain abdominal X-ray were the main imagistic methods of investigations. Preoperative resuscitation is paramount in the treatment of peritonitis in children. Analgesia, nutritional support and sometimes even transfusion are critical in the treatment result improvement. Percutaneous drainage is an option in localized fluid collections but in generalized peritonitis abdominal lavage with warm saline a drainage are always indicated. In our study we noticed that most of the patients in which we used targeted lavage with a rectal tube, the rate of complications improved. Basically we used a small rectal tube (10-16 Fr) which we oriented in all intraabdominal zones with difficult access like pelvic regions, sub hepatic and sub diaphragmatic regions. We used a Guyon syringe to wash the abdominal cavity trough the rectal tube. This proved to be very efficient in avoiding infectious and obstructive complications. Recently, little therapeutic progress has been made in the treatment of intra-abdominal infections. Regarding the antibiotic treatment, more and more antibiotics have been used, which is outstanding, but two molecules in particular prove of interest to us. Ertapenem is a carbapenem antibiotic with broad spectrum, resistant to beta-lactamase activity, but not as efficient against pseudomonas aeruginosa as imipenem and Meropenem. It is not used in any infections with pseudomonas aeruginosa. [2].Moxifloxacin has good peritoneum and gastrointestinal tract penetration and has been shown to be equally effective as levofloxacin combined with metronidazole in intraabdominal infections. It is given daily in one dose and it is a good therapeutic option for patients that are allergic to penicillin and betalactam antibiotics. Moxifloxacin does not require dose adjustment in patients with impaired renal function, giving clinicians a monotherapeutic option in the treatment of complicated IAI. [3]

\section{Conclusions}

Generalized acute peritonitis is defined as a severe and diffuse inflammation of the peritoneum, of bacterial or chemical origin. It represents a vital surgical emergency that requires hospitalization and urgent treatment.

This retrospective study on the 340 patients with generalized acute peritonitis allowed us to draw some important conclusions, regarding the objectives of the study:

- Generalized acute peritonitis occupies a special place in the abdominal emergencies in children by frequency and severity.

- The patient with generalized acute peritonitis corresponds to a particular epidemiological profile related to: low socio-economical level, young age, male gender and pathological background.

- The etiology of peritonitis remains varied, but are dominated by appendicular peritonitis.

- The prognostic of peritonitis depends on etiology, precociousness of diagnosis and treatment, age and associated conditions. 


\section{References}

1. Behrman RE, Kliegman RM, Jenson HB Acute

3. Brook I. Microbiology and management of abdominal secondary peritonitis. In: Nelson Textbook of infections. Dig Dis Sci 2008;53(10): 2585-91.

Pediatrics. (Eds). Philadelphia, Saunders, 2004, p 1352

2. Bratzler DW, Dellinger EP, Olsen KM et al (2013)

Clinical practice guidelines for antimicrobial prophylaxis in surgery.Surg Infect Larchmt 14:73-156. doi:10.1089/sur.2013.9999

\section{Correspondence to:}

Adrian Surd

Pediatric Surgery Department,

Emergency Children's Hospital,

Cluj-Napoca, Romania

Phone: +40740069239

Email: adisurd@yahoo.com 